\title{
Reverse-Engineering MAC: A Non-Cooperative Game Model
}

\author{
Jang-Won Lee, Member, IEEE, Ao Tang, Member, IEEE, Jianwei Huang, Member, IEEE, Mung \\ Chiang, Member, IEEE, and A. Robert Calderbank, Fellow, IEEE
}

\begin{abstract}
This paper reverse-engineers backoff-based random-access MAC protocols in ad-hoc networks. We show that the contention resolution algorithm in such protocols is implicitly participating in a non-cooperative game. Each link attempts to maximize a selfish local utility function, whose exact shape is reverse-engineered from the protocol description, through a stochastic subgradient method in which the link updates its persistence probability based on its transmission success or failure. We prove that existence of a Nash equilibrium is guaranteed in general. Then we establish the minimum amount of backoff aggressiveness needed, as a function of density of active users, for uniqueness of Nash equilibrium and convergence of the best response strategy. Convergence properties and connection with the best response strategy are also proved for variants of the stochastic-subgradient-based dynamics of the game. Together with known results in reverse-engineering TCP and BGP, this paper further advances the recent efforts in reverse-engineering layers 2-4 protocols. In contrast to the TCP reverse-engineering results in earlier literature, MAC reverse-engineering highlights the non-cooperative nature of random access.
\end{abstract}

Index Terms- Wireless network, Ad hoc network, Medium access control, Mathematical programming/optimization, Network utility maximization, Game theory, Network control by pricing, Reverse-engineering.

\section{INTRODUCTION}

$\mathbf{T}$ O BETTER understand backoff-based random-access protocols in wireless MAC (Medium Access Control), such as the BEB (Binary Exponential Backoff) protocol in the IEEE 802.11 DCF standard, we pose the following question: are the distributed and selfish actions by each link in such protocols in fact implicitly maximizing some local utility functions? We answer this question by developing a noncooperative game model for EB (Exponential Backoff) type of MAC protocols, reverse-engineering the underlying utility function's form from protocol description, and characterizing the existence, uniqueness, stability, and other key properties of Nash equilibrium.

Manuscript received July 1, 2006; revised February 15, 2007. This work was supported in part by NSF Grants CNS-0430487, CCF-0440443, CNS0417607, CCF-0448012, and CNS-0427677. Parts of the results have been presented at IEEE INFOCOM 2006 and IEEE WiOpt 2006.

J.-W. Lee is with the Department of Electrical and Electronic Engineering, Yonsei University, 134 Shinchon-dong, Seodaemun-gu, Seoul, Korea 120-749 (e-mail: jangwon@yonsei.ac.kr).

A. Tang is with the Social and Information Sciences Laboratory, Caltech, Pasadena, CA 91125, USA (e-mail: aotang@caltech.edu).

J. Huang, M. Chiang, and A. R. Calderbank are with the Department of Electrical Engineering, Princeton University, Princeton, NJ 08544, USA (e-mail: jianweih@princeton.edu, chiangm@princeton.edu, calderbk@princeton.edu).

Digital Object Identifier 10.1109/JSAC.2007.070808.
Reverse-engineering starts from a given protocol originally designed based on ad hoc heuristics and discovers the underlying mathematical problems implicitly being solved by the network dynamics of the given protocol. It does not proceed with a top-down design path, but often leads to new insights on why an existing protocol "works" and when it will not, thus indirectly leading to systematic forward-engineering.

The reverse-engineering approach in this paper is different from either imposing a particular utility maximization in a game-theoretic model (e.g., the game-theoretic model for slotted Aloha in [1]) or performance analysis of a protocol without discovering the underlying optimization process (e.g., analysis of 802.11 protocols based on Markov model [2], [3]). By studying the current MAC protocol, we discover that the users are actually implicitly participating a non-cooperative game, with the utility function of each selfish user (player in the game) reserve-engineered from the protocol specifications.

This reverse-engineering leads to a mathematical model to study the selfish behaviors in the current MAC protocol, including why certain aspects of the protocol work (e.g., uniqueness of the Nash Equilibrium can be shown), why others do not work (e.g., difficulty in attaining convergence and social optimality), and how to improve the MAC protocol by forward-engineering efforts (e.g., new MAC design [18] motivated by reverse-engineering). Another benefit of reverseengineering is that much insights on protocol performance and its cross-layer effects can be obtained. Our layer 2 reverseengineering results complement the recent success on reverseengineering layer 4 TCP, e.g., [5], [6], and layer 3 BGP [7].

It is interesting to note that reverse-engineering MAC reveals the non-cooperative nature of random access in contrast to TCP reverse-engineering results in recent research literature. Internet TCP/AQM protocols in the transport layer have recently been reverse-engineered as implicitly solving a cooperative Network Utility Maximization (NUM) [8], [9], [10], [11] using different Lagrange multipliers or congestion prices. Consider a communication network with $L$ logical links, each with a fixed capacity of $c_{l}$ bps, and $S$ sources (i.e., end users), each transmitting at a source rate of $x_{s}$ bps. Each source $s$ emits one flow, using a fixed set $L(s)$ of links in its path, and has a concave utility function $U_{s}\left(x_{s}\right)$. NUM is formulated as:

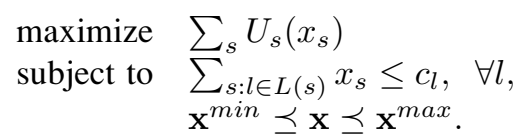

Even though TCP/AQM protocols were first designed without regard to global optimization, a reverse-engineering model 
provides a rigorous path towards understanding the equilibrium and dynamic properties of complicated interactions across sources and routers, as well as valuable guidance in design. In those models, the utility function of each source depends only on its data rate that can be directly controlled by the source itself, and there is adequate feedback from the network. Hence, the TCP/AQM protocol can be modeled as an algorithm that converges to the globally optimal rate allocation by implicitly solving the basic NUM problem (1) for different utility functions and its Lagrange dual problem.

Prior to this paper, there has not been a systematic effort of reverse-engineering existing MAC protocols. MAC protocols can be classified into two main categories: scheduling-based (contention free), e.g., FDMA, TDMA, CDMA, and random access (contention-based), e.g., Ethernet, slotted Aloha, 802.11 DCF function. Even though, recently, some different approaches are studied (e.g.[12]), scheduling-based MAC protocols have long been mapped into graph coloring or matching problems. Therefore, reverse-engineering is most necessary for the heuristics-based random access MAC protocols. These protocols typically include a contention avoidance phase and contention resolution phase. In the contention avoidance phase, users exchange messages (e.g., Request-ToSend (RTS) and Clear-To-Send (CTS)) to avoid simultaneous transmissions. In the contention resolution phase, the contention conflicts are resolved by various methods including adaptive persistence probability (e.g., slotted Aloha) and adaptive backoff window size (e.g., 802.11 DCF). In this paper, we will reverse-engineer an average model of protocols based on Exponential Backoff (EB).

Different from the TCP/AQM model, the utility of each link in the EB protocol directly depends on not just its own transmission (e.g., persistence probability) but also transmissions of other links due to collisions that cannot be controlled by the link itself. Moreover, there is no explicit feedback from the network. Hence, in contrast to TCP reverse engineering, a non-cooperative game model is more appropriate for the EB protocol than a global optimization model. We show that the EB protocol can be reverse-engineered through a non-cooperative game in which each link tries to maximize, using a stochastic subgradient formed by local information, its own utility function in the form of expected net reward for successful transmission. While the existence of Nash equilibrium can be proved, neither convergence nor social welfare optimality is guaranteed. We then provide sufficient conditions on link density and backoff aggressiveness that guarantee uniqueness and stability (i.e., convergence of the standard best response strategy as well as the gradient update with small step size) of Nash equilibrium. In particular, it confirms and quantifies the intuition that as long as there is adequate backoff among contending nodes, the non-cooperative game dynamics of random access will converge. Finally we show that a sequential variant of the stochastic subgradient method is equivalent to the best response strategy.

The rest of this paper is organized as follows. In Section II, we provide the system model. In Section III-A, we establish a non-cooperative game model for the EB protocol, reverseengineer the underlying utility function, and prove the existence of Nash equilibrium. In Section III-B, we further reverse-

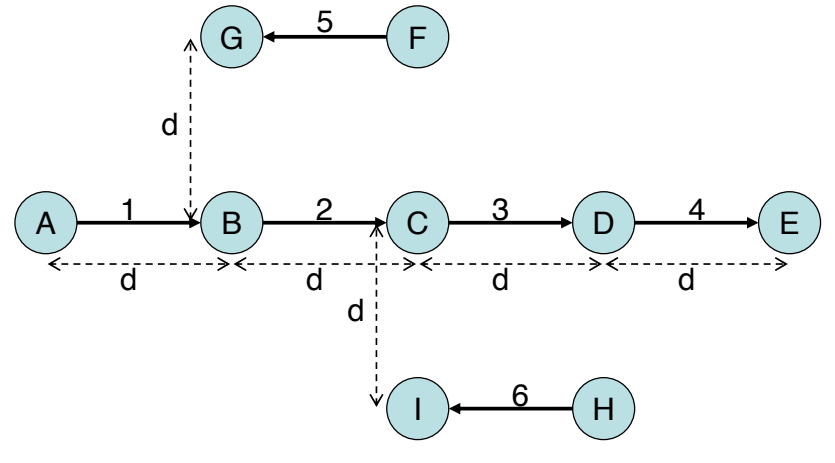

Fig. 1. Logical topology graph of an example network.

engineer the EB protocol as a stochastic subgradient method. We characterize the uniqueness and stability properties of Nash equilibrium in Sections III-C and III-D, and develop the relationship between the stochastic subgradient method and the best response strategy in Section III-E. In Section IV, we provide numerical results that illustrate the properties of the EB protocol as a non-cooperative game, and we conclude in Section V. Most of the proofs are presented in the Appendix.

\section{System Model}

Consider an ad-hoc network represented by a directed graph $G(V, E), e . g$., as in Figure 1, where $V$ is the set of nodes and $E$ is the set of logical links. We define $L_{t o}^{I}(l)$ as the set of links whose transmissions cause interference to the receiver of link $l$ and $L_{\text {from }}^{I}(l)$ as the set of links whose transmissions get interfered from the transmission of link $l$. Hence, if link $l$ and a link in set $L_{t o}^{I}(l)$ transmit data simultaneously, the transmission of link $l$ fails. If link $l$ and a link $k$ in set $L_{\text {from }}^{I}(l)$ transmit data simultaneously, the transmission of link $k$ also fails.

The EB protocol is a prototypical contention resolution protocol in such wireless networks. EB protocols can be implemented in two ways: a persistence-probability-based protocols and contention-window-based protocols similar to slotted Aloha. In the persistence probability-based EB protocol, each link $l$ has its own persistence probability $p_{l}$ with which it transmits its data in a time-slot, and the maximum and minimum persistence probabilities $p_{l}^{\max }$ and $p_{l}^{\min }$. After each transmission attempt, if the transmission is successful without collisions, then link $l$ sets its persistence probability to be its maximum value, $p_{l}^{\max }$. Otherwise, it multiplicatively reduces its persistence probability by a factor $\beta_{l}\left(0<\beta_{l}<1\right)$ until reaching its minimum value $p_{l}^{\min }$. In the contention windowbased EB protocol, each link $l$ maintains its contention window size $W_{l}$, current window size $C W_{l}$, and minimum and maximum window sizes $W_{l}^{\min }$ and $W_{l}^{\max }$. After each transmission, contention window size and current window size are updated. If transmission is successful, the contention window size is reduced to the minimum window size (i.e., $\left.W_{l}=W_{l}^{\text {min }}\right)$, otherwise it is increased by a factor $1 / \beta_{l}$ $\left(0<\beta_{l}<1\right)$ until reaching the maximum window size $W_{l}^{\max }$ (i.e., $W_{l}=\min \left\{1 / \beta_{l} W_{l}, W_{l}^{\max }\right\}$ ). Then, current window size $C W_{l}$ is updated to be a number between $\left(0, W_{l}\right)$ following a uniform distribution. It decreases in every time-slot, and when it becomes zero, the link transmits data. 
In the IEEE 802.11 implementation, the EB protocol is window-based with $\beta=1 / 2$. Since the window size is doubled after each transmission failure, the EB protocol in the IEEE 802.11 is called the Binary Exponential Backoff (BEB) protocol, which is a special case of EB protocols.

Here we study the persistence probability-based EB protocol, which can also approximate a contention windowbased EB protocol, just like the source rate model in the TCP reverse-engineering literature for the window-based TCP congestion control protocol. The correspondence can be seen by setting $p_{l}=2 /\left(W_{l}+1\right), p_{l}^{\max }=2 /\left(W_{l}^{\text {min }}+1\right)$, and $p_{l}^{\text {min }}=2 /\left(W_{l}^{\max }+1\right)$. This is justified due to the following reasons. First, after the transmission, the time until the next transmission has a geometric distribution with a parameter $p_{l}$ (i.e., with mean $1 / p_{l}$ ) in the persistence probability-based protocol and a uniform distribution between 1 and $W_{l}$ (i.e., with mean $\left.\left(W_{l}+1\right) / 2\right)$ in the contention window-based protocol. Hence, with the above relationship between the persistence probability and the window size, both protocols have the same mean value for the inter-transmission time. Moreover, it has been shown in [2] that the above relationship is valid once the window size (or the persistence probability) converges to the equilibrium point.

\section{REVERSE-ENGINEERING: NON-COOPERATIVE GAME MOdEL OF EB MAC PROTOCOL}

In this section, we characterize the selfish utility maximization problem that is implicitly solved by random-access MAC protocols such as EB. In contrast to the TCP/AQM protocol that can be modeled as a basic NUM in (1), we model the EB protocol as a non-cooperative game due to the coupled utility of each link (due to collisions) and the lack of sufficient feedback from the network.

\section{A. Game Model, Utility Function, and Existence of Nash Equilibrium}

The update algorithm for the persistence probability described in the previous section can be written as:

$$
\begin{aligned}
p_{l}(t+1)= & \max \left\{p_{l}^{\min }, p_{l}^{\max } \mathbf{1}_{\left\{T_{l}(t)=1\right\}} \mathbf{1}_{\left\{C_{l}(t)=0\right\}}\right. \\
& \left.+\beta_{l} p_{l}(t) \mathbf{1}_{\left\{T_{l}(t)=1\right\}} \mathbf{1}_{\left\{C_{l}(t)=1\right\}}+p_{l}(t) \mathbf{1}_{\left\{T_{l}(t)=0\right\}}\right\},
\end{aligned}
$$

where $p_{l}(t)$ is a persistence probability of link $l$ at time-slot $t, \mathbf{1}_{a}$ is an indicator function of event $a$, and $T_{l}(t)$ and $C_{l}(t)$ are the events that link $l$ transmits data at time-slot $t$ and that there is a collision to link $l$ 's transmission given that link $l$ transmits data at time-slot $t$, respectively. Then, given $\mathbf{p}(t)$, we have

$$
\operatorname{Prob}\left\{T_{l}(t)=1 \mid \mathbf{p}(t)\right\}=p_{l}(t)
$$

and

$$
\operatorname{Prob}\left\{C_{l}(t)=1 \mid \mathbf{p}(t)\right\}=1-\prod_{n \in L_{t o}^{I}(l)}\left(1-p_{n}(t)\right) .
$$

Since the update of the persistence probabilities for the next time-slot depends only on the current persistence probabilities, we will consider the update conditioning on the current persistence probabilities. Note that $p_{l}(t)$ is a random process whose transitions depend on events $T_{l}(t)$ and $C_{l}(t)$.
We will first study its expected (thus deterministic) trajectory, and will return to (2) later in this section ${ }^{1}$. Slightly abusing the notation, we still use $p_{l}(t)$ to denote the expected persistence probability. From (2), we have

$$
\begin{aligned}
p_{l}(t+1)= & \max \left\{p_{l}^{\min }, p_{l}^{\max } \mathrm{E}\left\{\mathbf{1}_{\left\{T_{l}(t)=1\right\}} \mathbf{1}_{\left\{C_{l}(t)=0\right\}} \mid \mathbf{p}(t)\right\}\right. \\
& +\beta_{l} \mathrm{E}\left\{p_{l}(t) \mathbf{1}_{\left\{T_{l}(t)=1\right\}} \mathbf{1}_{\left\{C_{l}(t)=1\right\}} \mid \mathbf{p}(t)\right\} \\
& \left.+\mathrm{E}\left\{p_{l}(t) \mathbf{1}_{\left\{T_{l}(t)=0\right\}} \mid \mathbf{p}(t)\right\}\right\} \\
= & \max \left\{p_{l}^{\text {min }}, p_{l}^{\text {max }} p_{l}(t) \prod_{n \in L_{t o}^{I}(l)}\left(1-p_{n}(t)\right)\right. \\
& +\beta_{l} p_{l}(t) p_{l}(t)\left(1-\prod_{n \in L_{t o}^{I}(l)}\left(1-p_{n}(t)\right)\right) \\
& \left.+p_{l}(t)\left(1-p_{l}(t)\right)\right\},
\end{aligned}
$$

where $\mathrm{E}\{a \mid b\}$ is the expected value of $a$ given $b$.

We now reverse-engineer the update algorithm in (3) as a game, in which each link $l$ updates its strategy, i.e., its persistence probability $p_{l}$, to maximize its utility $U_{l}$ based on strategies of the other links, i.e., $\mathbf{p}_{-l}=$ $\left(p_{1}, \cdots, p_{l-1}, p_{l+1}, \cdots, p_{|E|}\right)$.

Formally, we formulate the EB protocol as a noncooperative game, $G_{E B-M A C}=\left[E, \times_{l \in E} A_{l},\left\{U_{l}\right\}_{l \in E}\right]$, where $E$ is a set of players, i.e., links, $A_{l}=\left\{p_{l} \mid p_{l}^{\text {min }} \leq\right.$ $\left.p_{l} \leq p_{l}^{\max }\right\}$ is an action set of player $l$, and $U_{l}$ is a utility function of player $l$. We refer to this as the EB-MAC Game and now study its properties and solutions.

In the non-cooperative game, one of the most important questions is whether a Nash equilibrium ${ }^{2}$ [13] exists or not. In the case of EB-MAC Game, we have the following definition of Nash equilibrium.

Definition 1: A persistence probability vector $\mathbf{p}^{*}$ is said to be a Nash equilibrium if no link can improve its utility by unilaterally deviating its persistence probability from Nash equilibrium:

$$
U_{l}\left(p_{l}^{*}, \mathbf{p}_{-l}^{*}\right) \geq U_{l}\left(p_{l}, \mathbf{p}_{-l}^{*}\right), p_{l}^{\min } \leq p_{l} \leq p_{l}^{\max }, \quad \forall l .
$$

The following reverse-engineering theorem, proved in Appendix A, obtains the underlying utility functions in the EBMAC Game and establishes the existence of Nash equilibrium for the game.

Theorem 1: The utility function is the following expected net reward (expected reward minus expected cost) that the link can obtain from its transmission:

$$
\begin{aligned}
& U_{l}(\mathbf{p})=p_{l}^{2}\left(\frac{1}{2} p_{l}^{\max }-\frac{1}{3} p_{l}\right) \prod_{n \in L_{t o}^{I}(l)}\left(1-p_{n}\right) \\
& -\frac{1}{3}\left(1-\beta_{l}\right) p_{l}^{3}\left(1-\prod_{n \in L_{t o}^{I}(l)}\left(1-p_{n}\right)\right) \\
& =R\left(p_{l}\right) S(\mathbf{p})-C\left(p_{l}\right) F(\mathbf{p}), \quad \forall l,
\end{aligned}
$$

${ }^{1}$ To be precise, the expectation of $p_{l}(t)$ needs to be taken outside of "max", instead of inside "max" as in (3). However, if we make $p_{l}^{\min }=0$, as we will do later in the paper, the "max" operation is not needed and (3) yields the precise average value. Moreover, in most practice cases, $p_{l}^{\min } \approx 0$ and (3) can be a good approximation.

${ }^{2}$ In this paper, we only consider a pure Nash equilibrium as in Definition 1. 


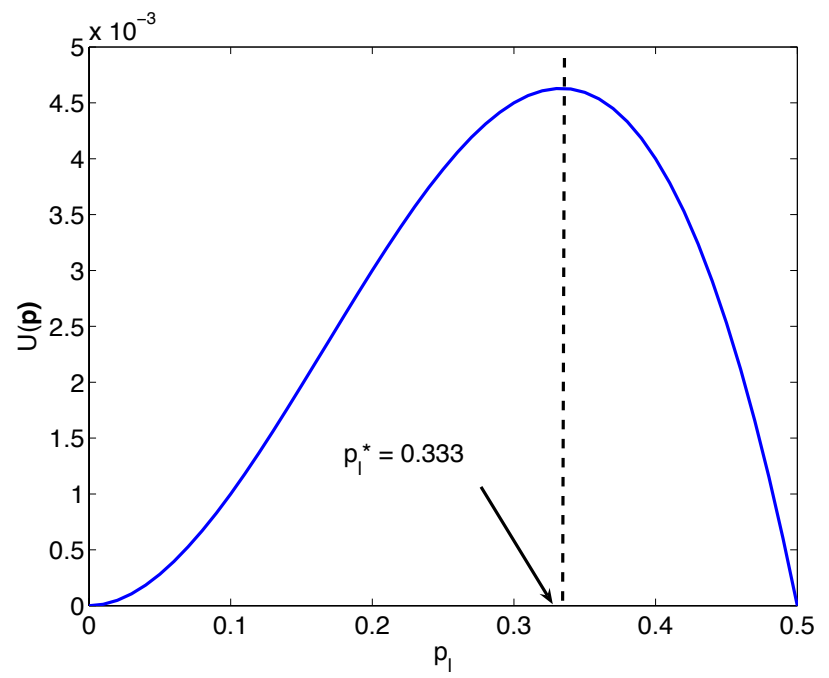

Fig. 2. Dependence of a utility function on its own persistence probability, for $\beta_{l}=0.5, p_{l}^{\max }=0.5$, and $\prod_{n \in L_{t o}^{I}(l)}\left(1-p_{n}\right)=0.5$.

where $S(\mathbf{p})=p_{l} \prod_{n \in L_{t o}^{I}(l)}\left(1-p_{n}\right)$ is the probability of transmission success, $F(\mathbf{p}) \stackrel{=}{=} p_{l}\left(1-\prod_{n \in L_{t o}^{I}(l)}\left(1-p_{n}\right)\right)$ is the probability of transmission failure, and $R\left(p_{l}\right) \stackrel{\text { def }}{=} p_{l}\left(\frac{1}{2} p_{l}^{\max }-\right.$ $\left.\frac{1}{3} p_{l}\right)$ can be interpreted as the reward for transmission success, $C\left(p_{l}\right) \stackrel{\text { def }}{=} \frac{1}{3}\left(1-\beta_{l}\right) p_{l}^{2}$ can be interpreted as the cost for transmission failure. Furthermore, there exists a Nash equilibrium in the EB-MAC Game $G_{E B-M A C}=\left[E, \times_{l \in E} A_{l},\left\{U_{l}\right\}_{l \in E}\right]$ characterized by the following:

$$
p_{l}^{*}=\frac{p_{l}^{\max } \prod_{n \in L_{t o}^{I}(l)}\left(1-p_{n}^{*}\right)}{1-\beta_{l}\left(1-\prod_{n \in L_{t o}^{I}(l)}\left(1-p_{n}^{*}\right)\right)}, \quad \forall l .
$$

Remark: It is important to note that the expressions of $S(\mathbf{p})$ and $F(\mathbf{p})$ come directly from the definitions of success and failure probabilities, while the expressions of $R\left(p_{l}\right)$ and $C\left(p_{l}\right)$ (thus exact form of $U_{l}$ ) are in fact derived in the proof by reverse-engineering the EB protocol description.

From (5), we conclude that, other conditions being the same, at a Nash Equilibrium a link $l$ will have a higher persistence probability if it has a higher value of $p_{l}^{\max }$, a higher value of $\beta_{l}$, or a higher value of $\prod_{n \in L_{\text {to }}^{I}(l)}\left(1-p_{n}^{*}\right)$, i.e., a higher transmission success probability. We also have the next corollaries that immediately follow from (3) and (5).

Corollary 1: If $\mathbf{p}(t)$ updated by (3) converges to $\mathbf{p}^{*}$, $\mathbf{p}^{\min }<\mathbf{p}^{*}<\mathbf{p}^{\max }$, then $\mathbf{p}^{*}$ is a Nash equilibrium.

Corollary 2: Suppose that $p_{l}^{\text {min }}>0, \forall l, p_{l}^{*} \rightarrow$ $p_{l}^{\text {min }}$ as $\left|L_{t o}^{I}(l)\right| \rightarrow \infty$.

Corollary 3: Suppose that $p_{l}^{\text {min }}=0, \forall l$. Let $\left|L_{t o}^{I}(l)\right| \rightarrow$ $\infty$. If $p_{l}^{*}>0$, then only a finite number of links among links in $L_{t o}^{I}(l)$ have positive persistence probabilities at a Nash equilibrium.

Corollaries 2 and 3 can be easily proven with (14) and the fact that, as the number of links in $L_{t o}^{I}(l)$ with a positive persistence probability at a Nash equilibrium goes to infinity, $p_{l}^{*}$ in (5) goes to zero. Corollaries 2 and 3 confirm the intuition that, as the number of interfering nodes to a link increases (i.e., as the amount of contention in the contention region of a link gets higher), the persistence probability of the link decreases.

\section{B. EB Protocol and Stochastic Subgradient Method}

Using (12), we can rewrite (3) as

$$
p_{l}(t+1)=\max \left\{p_{l}^{\text {min }}, p_{l}(t)+\left.\frac{\partial U_{l}(\mathbf{p})}{\partial p_{l}}\right|_{\mathbf{p}=\mathbf{p}(t)}\right\} \text {. }
$$

Hence, in (3), each link updates its persistence probability to the direction of the maximizer using the gradient. To update its persistence probability by (3), each link $l$ must know the persistence probabilities of its adjacent links, i.e., link $n, n \in L_{t o}^{I}(l)$. However, in the EB protocol, there is no explicit message passing among links, and the link cannot obtain the exact information to evaluate the gradient of its utility function. Instead of using the exact gradient of its utility function as in (3), each link attempts to approximate it using (2) according to a stochastic subgradient method as defined follows [14].

Definition 2: Consider a function $g(x)$ and a sequence of random variables $(x(0), \ldots, x(t))$ from time 0 to $t$. Then $f(t)$ is said to be a stochastic subgradient of $g(x)$ at $x(t)$ if

$$
\mathrm{E}[f(t) \mid(x(0), \ldots, x(t))] \in \partial g(x),
$$

where $\partial g(x)$ is the subdifferential, i.e., the set of subgradients of $g(x)$.

Hence, the stochastic subgradient of a function can be thought as the perturbed version of the subgradient of the function (i.e., gradient, when the gradient exists) whose conditional expected value is equal to the subgradient.

We now rewrite (2) as

$$
\begin{aligned}
p_{l}(t+1)= & \max \left\{p_{l}^{\min }, p_{l}(t)-p_{l}(t)+p_{l}^{\max } \mathbf{1}_{\left\{T_{l}(t)=1\right\}} \mathbf{1}_{\left\{C_{l}(t)=0\right\}}\right. \\
& \left.+\beta_{l} p_{l}(t) \mathbf{1}_{\left\{T_{l}(t)=1\right\}} \mathbf{1}_{\left\{C_{l}(t)=1\right\}}+p_{l}(t) \mathbf{1}_{\left\{T_{l}(t)=0\right\}}\right\} \\
= & \max \left\{p_{l}^{\min }, p_{l}(t)+v_{l}(t)\right\},
\end{aligned}
$$

where

$$
\begin{aligned}
v_{l}(t)= & p_{l}^{\max } \mathbf{1}_{\left\{T_{l}(t)=1\right\}} \mathbf{1}_{\left\{C_{l}(t)=0\right\}}+\beta_{l} p_{l}(t) \mathbf{1}_{\left\{T_{l}(t)=1\right\}} \mathbf{1}_{\left\{C_{l}(t)=1\right\}} \\
& +p_{l}(t) \mathbf{1}_{\left\{T_{l}(t)=0\right\}}-p_{l}(t) .
\end{aligned}
$$

Since

$$
\begin{aligned}
\mathrm{E}\left\{v_{l}(t) \mid \mathbf{p}(t)\right\}= & p_{l}^{\max } p_{l}(t) \prod_{n \in L_{t o}^{I}(l)}\left(1-p_{n}(t)\right) \\
& +\beta_{l} p_{l}(t) p_{l}(t)\left(1-\prod_{n \in L_{t o}^{I}(l)}\left(1-p_{n}(t)\right)\right) \\
& +p_{l}(t)\left(1-p_{l}(t)\right)-p_{l}(t) \\
= & \left.\frac{\partial U_{l}(\mathbf{p})}{\partial p_{l}}\right|_{\mathbf{p}=\mathbf{p}(t)},
\end{aligned}
$$

we conclude that $v_{l}(t)$ is a stochastic subgradient of $U_{l}$ at $\mathbf{p}(t)$.

In summary, we have the following reverse-engineering result in addition to Theorem 1:

Theorem 2: The EB protocol described by (2) is a stochastic subgradient algorithm to maximize utility in (4).

Remark: Each stochastic subgradient $v_{l}$ can be measured by the link itself through collision and success of its transmission, without explicit message passing among links. 


\section{Uniqueness of Nash Equilibrium and Convergence of Best Response}

In Theorem 1, we have shown that there exists a Nash equilibrium in the EB-MAC game. However, in general, there may not be a unique Nash equilibrium, as illustrated in a simple example. Suppose that there are two links interfering with each other, and that $p_{1}^{\max }=p_{2}^{\max }=p^{\max }=1$, then it can be verified that there is an infinite number of Nash equilibria, which is the set of $\left(p_{1}^{*}, p_{2}^{*}\right)$ satisfying

$$
\max \left\{p^{\min }, \frac{1-p^{\max }}{1-\beta p^{\max }}\right\} \leq p_{1}^{*} \leq \min \left\{1, \frac{1-p^{\min }}{1-\beta p^{\min }}\right\}
$$

and

$$
p_{2}^{*}=\frac{1-p_{1}^{*}}{1-\beta p_{1}^{*}} .
$$

We will investigate uniqueness of Nash equilibrium together with the convergence of a natural strategy for the game: the best response strategy, commonly used to study stability of Nash equilibrium.

Definition 3: Link $l$ 's best response is defined as the persistent probability that maximizes his utility function given fixed persistent probabilities from other links:

$$
\mathcal{B}_{l}\left(\mathbf{p}_{-l}\right)=\underset{p_{l}^{\text {min }} \leq p_{l} \leq p_{l}^{\text {max }}}{\operatorname{argmax}} U_{l}\left(p_{l}, \mathbf{p}_{-l}\right) .
$$

Thus the best response update is defined as

$$
p_{l}^{*}(t+1)=\mathcal{B}_{l}\left(\mathbf{p}_{-l}^{*}(t)\right), \forall l \text {. }
$$

Note that, in current practice, the persistence probability in the EB protocol is not updated by the best response strategy, but by (2) (or by (3) on average). Hence, in the EB protocol, instead of instantaneously setting $p_{l}(t+1)$ to the best response $p_{l}^{*}(t+1)$, in (2) (or (3)) each link updates its persistence probability to the direction of the maximizer by using the stochastic gradient. Hence, in the EB protocol, the persistence probability of the link is updated more smoothly than the best response.

As proved in Appendix B based on S-modular game theory [15], [16], the following theorem provides our first characterization of the convergence properties of the best response strategy to a Nash equilibrium in the EB-MAC Game.

Theorem 3: Suppose that the persistence probability of each link is updated by the best response function in (6) in each time-slot with $\mathbf{p}^{*}(0)=\mathbf{p}^{\text {min }}$. Then,

$$
\mathbf{p}^{*}(2 t+1) \rightarrow \hat{\mathbf{p}} \text { and } \mathbf{p}^{*}(2 t) \rightarrow \tilde{\mathbf{p}} \text { as } t \rightarrow \infty
$$

If $\hat{\mathbf{p}}=\tilde{\mathbf{p}}$ i.e., if $\mathbf{p}^{*}(t)$ converges to $\hat{\mathbf{p}}$, then $\hat{\mathbf{p}}$ is a Nash equilibrium.

Thus far, we have shown that Nash equilibrium of the EBMAC game may not be unique and, further, the best response strategy may not converge to a Nash equilibrium. However, by imposing some conditions on the strategy set of each link, we can guarantee both uniqueness of Nash equilibrium and convergence of the best response strategy to the Nash equilibrium.

For notational simplicity, we assume all links have the same $p^{\max }$ and $p^{\min }$. Furthermore, assume that $p^{\max }<1$ and $p^{\min }=0^{3}$. Then, from (5), we have

$$
p_{l}^{*}=p^{\max } \frac{\prod_{n \in L_{t o}^{I}(l)}\left(1-p_{n}^{*}\right)}{1-\beta\left(1-\prod_{n \in L_{t o}^{I}(l)}\left(1-p_{n}^{*}\right)\right)},
$$

where $L_{t o}^{I}(l)$ is a set of links that cause interference to link $l$. We first bound Nash equilibrium with the following

Lemma 1: We have $p_{l}^{*}>0$ and $p_{l}^{*}<p^{\max }$.

This lemma is proved in Appendix $\mathrm{C}$ and guarantees that any equilibrium must be an inner solution. We now show that when contention density is not too high, the above solution is actually the unique Nash equilibrium.

Let $K=\max _{l}\left\{\left|L_{t o}^{I}(l)\right|\right\}$, which captures the amount of potential contention among links. We have the following theorem that relates three key quantities: amount of potential contention $K$, backoff multiplier $\beta$ (speed of backoff), and $p^{\max }$ that corresponds to the minimum contention window size (minimum amount of backoff).

Theorem 4: If $\frac{p^{\max } K}{4 \beta\left(1-p^{\max }\right)}<1$, then

1) The Nash equilibrium is unique;

2) Start from any initial point, the iteration defined by best response converges to the unique equilibrium.

The proof is in Appendix D. The key idea is to show the updating rule from $p(t)$ to $p(t+1)$ is a contraction mapping [17] by verifying a particular norm of the Jacobian $\mathbf{J}\left(\|\mathbf{J}\|_{\infty}\right.$ in our proof) is less than one.

There are several interesting engineering implications from the above theorem. For example, it provides a guidance to choose parameter in EB protocols, and quantifies the intuition that with a large enough $\beta$ (i.e., links do not decrease the probabilities suddenly) and a small enough $p^{\max }$ (i.e., links backoff aggressively enough), uniqueness and stability can be ensured. The higher the amount of contention (i.e., a larger value of $K$ ), the smaller $p^{\max }$ needs to be.

Some of the other implications are stated in the following corollary, whose proof hinges upon the following observation. If $\beta \leq 0.5$, then $\frac{1-\beta}{\beta(1-p)} \geq 1$ for $p \in(0,1)$, and we have

$$
\begin{aligned}
\|\mathbf{J}\|_{\infty} & \leq \max _{l}\left\{\frac{p^{\max }\left|L_{t o}^{I}(l)\right|(1-\beta)}{\left(1-\beta+\beta\left(1-p^{\max }\right)\right)^{2}}\right\} \\
& \leq \frac{p^{\max } K(1-\beta)}{\left(1-\beta+\beta\left(1-p^{\max }\right)\right)^{2}} .
\end{aligned}
$$

Corollary 4: If one of the following conditions is satisfied, then the Nash equilibrium is unique. Moreover, starting from any initial point, the iteration defined by best response converges to the unique equilibrium.

(a) $\beta \leq 0.5$ and $\frac{p^{\max } K(1-\beta)}{\left(1-\beta+\beta\left(1-p^{\max }\right)\right)^{2}}<1$;

(b) For the system in which each link interferes each other (i.e., $\left.L_{t_{o}}^{I}(l)=E-\{l\}, \forall l\right)$, e.g., as in an uplink topology $\mathrm{y}^{4}, \frac{p^{\text {max }}(L-1)}{4 \beta\left(1-p^{\text {max }}\right)}<1$, where $L$ is the number of links;

(c) For the system in which each link interferes each other (i.e., $\left.L_{\text {to }}^{I}(l)=E-\{l\}, \forall l\right), \beta \leq 0.5$ and $\frac{p^{\max }(L-1)(1-\beta)}{\left(1-\beta+\beta\left(1-p^{\max }\right)\right)^{2}}<1$.

${ }^{3}$ If the maximum window size is sufficiently large, then $p^{\text {min }}$ can be sufficient close to 0 . Furthermore, if we do not allow the minimum window to be 1 , which is a plausible thing to do, then the smallest minimum window is 2 and the corresponding $p^{\max }=2 / 3<1$.

${ }^{4}$ Multiple users communicating with base station or access point with one hop. 


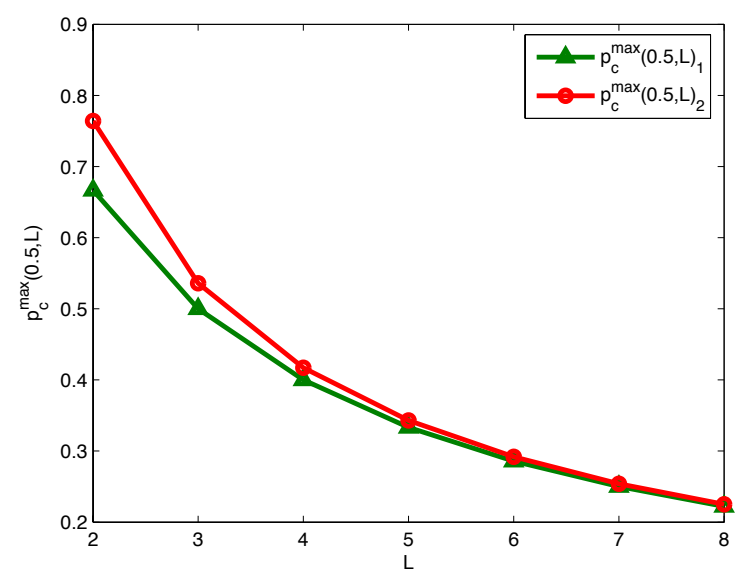

Fig. 3. $p_{c}^{\max }(\beta, L)$ for $\beta=0.5$.

Remark: Part (c) of the above corollary quantifies the intuition that smaller number of interfering links helps uniqueness and stability of Nash equilibrium: $L$ needs to be smaller than $1+\frac{\left(1-\beta+\beta\left(1-p^{\max }\right)\right)^{2}}{p^{\max }(1-\beta)}$.

Interpreting the above results in another way, we examine the dependence of the maximum $p^{\max }$ allowed, i.e., the least amount of backoff needed in terms of the smallest $W^{\text {min }}$, in order to ensure uniqueness and stability of EB protocol, as a function of backoff multiplier $\beta$ and link density $L$. Using $p_{c}^{\max }(\beta, L)$ to denote the critical value of $p^{\max }$ satisfying the bounds, both $p_{c}^{\max }(\beta, L)$ developed in Corollaries 4 (b) $\left(p_{c}^{\max }(\beta, L)_{1}\right)$ and 4 (c) $\left(p_{c}^{\max }(\beta, L)_{2}\right)$ are visualized in Figure 3 with the standard parameter $\beta=0.5$. It is worthwhile to note that as long as the minimum window size is 3 or larger, then for the number of active links $L$ up to 8 , which is a reasonably large number in many applications, uniqueness and convergence can be guaranteed.

We also plot $p_{c}^{\max }(\beta, L)$ for Corollary 4 (b) in Figure 4. Not surprisingly, $p_{c}^{\max }(\beta, L)$ in an increasing function on $\beta$ and decreasing on $L$. Moreover, it is concave on $\beta$ and convex on $L .5$

\section{Convergence of Gradient Play with Small Stepsize}

In the previous subsection we have shown the uniqueness of Nash equilibrium and convergence of the best response updates. In this subsection, we will show that an improved version of the gradient play in (3) also have nice convergence results.

Define the gradient play with small stepsize as

$$
p_{l}(t+1)=\max \left\{p_{l}^{\min }, p_{l}(t)+\kappa \frac{\partial U_{l}(\mathbf{p})}{\partial p_{l}}\right\},
$$

where $\partial U_{l}(\mathbf{p}) / \partial p_{l}$ is given in (12), and $\kappa$ is a constant stepsize no larger than 1 to ensure that $p_{l}(t+1) \leq p_{l}^{\max }$. It is clear

\footnotetext{
${ }^{5}$ A natural question to ask next is whether the above upperbounds on $p_{l}^{\max }$ are too conservative due to relaxations during the computation of bounds on Jacobian's infinity norm. The answer is no, for the contraction mapping technique used above. An limit that sets the best possible upperbound we can achieve via contraction mapping using infinity norm is derived by finding the lowerbound of the maximum of $\|J\|_{\infty}$, see Appendix E for details. It turns out this limit has qualitatively the same shape as the bounds in Corollary 4 .
}

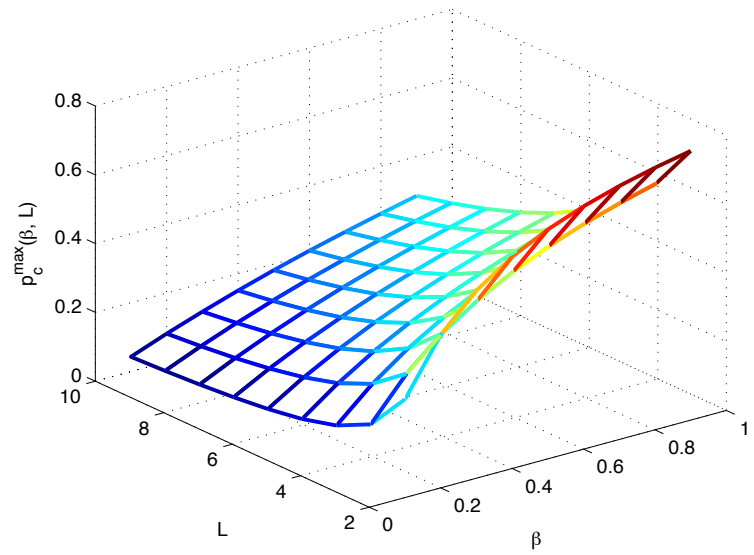

Fig. 4. $p_{c}^{\max }(\beta, L)$ from Corollary 4 (b).

that any fixed point of (9) is a Nash equilibrium. In (9), users update their persistent probabilities along the directions that improve their utility, which can be thought as better responses instead of best responses. If the $\kappa$ is small enough, (9) will converge to the Nash equilibrium and have less oscillation than the best response updates.

In the following we assume that each link $l$ interferes with each other (i.e., $L_{t o}^{I}(l)=E-\{l\}$ ), and $p_{l}^{\min }>0$ for all $l$. Let $\gamma=\frac{1}{\min _{l}\left(1-\beta_{l}\right) p_{l}^{\max }}$, and then we can prove the following:

Theorem 5: Assume there exists a unique Nash equilibrium. Then (9) globally converges to the unique Nash equilibrium if for any $l \in E$, stepsize $\kappa \leq$ $\min \left\{1, \frac{2}{\max _{l}\left(p_{l}^{\max }\right)^{2}(\gamma+|E|-1)}\right\}$ for any $p_{l} \in\left[p_{l}^{\min }, p_{l}^{\max }\right]$.

The proof is given in Appendix F. The basic idea is to define a Lyapunov function and show that it will keep increasing over time until (9) hits its fixed point, which is the Nash equilibrium.

Remark: Theorem 5 quantifies the intuition that the stepsize should decrease with a larger number of interfering links $|E|$, a larger value of $\max _{l}\left(p_{l}^{\max }\right)$, or a larger value of $\min _{l} \beta_{l}$. In other words, $\kappa$ should be chosen such that the total change in $\left(p_{l}(t+1)-p_{l}(t)\right)$ is small for all link $l$.

\section{E. Relating Stochastic Subgradient Method with Best Re- sponse Strategy}

We have shown that the stochastic subgradient updates (2) is how EB protocol works. A different update rule, the best response strategy (6), is the standard game-theoretic dynamics whose convergence characterizes the stability of Nash equilibrium, and we have provided sufficient conditions for its convergence. In this subsection, we develop the connection between these two types of updates.

Consider the case where only link $l$ updates its persistent probability $p_{l}$ similar to (2) but with a diminishing step-size, and other links contend for the common channel with fixed probabilities $\mathbf{p}_{-l}$. We can show that such sequential stochastic subgradient updates converge to the best response solution in (6) under proper chosen step-size and mild conditions of the system parameters. 


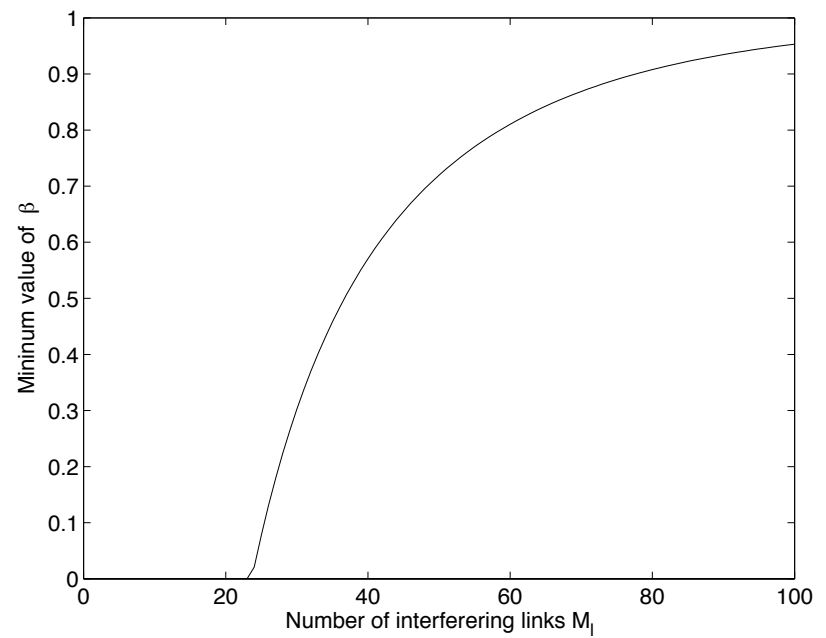

Fig. 5. The minimum value of $\beta$ that satisfies condition 3 of Theorem 6 vs. the number of interfering links $M_{l}$ with an infrared physical layer in 802.11 [2]

Formally, define the new update algorithm for link $l$ under fixed value of $\mathbf{p}_{-l}$ as:

$$
p_{l}(t+1)=\max \left\{\tilde{p}_{l}^{\min }, p_{l}(t)+\kappa(t) v_{l}(t)\right\},
$$

where $v_{l}(t)$ is the stochastic subgradient defined in (6), $\kappa(t)$ is the step-size (no larger than 1 ), and $\tilde{p}_{l}^{\min }$ is the modified minimum persistent probability. Assume for simplicity that all links have the same minimum and maximum persistent probabilities $0 \leq p^{\min } \leq p^{\max }<1$, and a common backoff multiplier $\beta$. The following result is proved in Appendix G.

Theorem 6: The update in (10) converges, with probability one, to the best response solution of link $l$ in (6) under fixed $\mathbf{p}_{-l}$ if the following conditions all hold:

1) The step-size $\kappa(t)$ satisfies $\kappa(t) \geq 0, \sum_{t=0}^{\infty} \kappa(t)=$ $\infty, \sum_{t=0}^{\infty} \kappa^{2}(t)<\infty$, e.g., $\kappa(t)=1 / t$.

2) The modified minimum persistent probability $\tilde{p}_{l}^{\min }=$ $\frac{p^{\max }\left(1-p^{\min }\right)^{M_{l}}}{1-\beta\left(1-\left(1-p^{\min }\right)^{M_{l}}\right)} \geq p^{\min }$

3) The values of $p^{\min }, p^{\max }$ and $\beta$ satisfy $\frac{1-\beta}{\beta}\left(\frac{1}{\left(1-p^{\max }\right)^{M_{l}}}-\frac{2}{\left(1-p^{\min }\right)^{M_{l}}}\right) \leq 1$, where $M_{l}=\left|L_{t o}^{I}(l)\right|$ is the number of interfering links with link $l$.

Remark: Theorem 6 shows that although link $l$ neither knows the exact values of other links' persistent probabilities, nor has memory of other links' past behaviors, the stochastic subgradient updates can still converge to the best response strategy, if it is sequential and use diminishing step-sizes (condition 1 above).

We now show that conditions 2 and 3 in Theorem 6 are often satisfied in practice. Both are on system parameters: the upperbound constraint on $p^{\mathrm{min}}$ in condition 2 , and the relationship in condition 3. If $p^{\mathrm{min}}=0$ as assumed in Section III-C, then condition 2 always holds, and a sufficient condition for condition 3 to hold is $2\left(1-p^{\max }\right)^{M_{l}} \geq 1$.

To see how often conditions 2 and 3 hold in practice, consider the system parameters specified in 802.11 standard (e.g., [2]). For an infrared (IR) physical layer, the minimum and maximum contention window sizes are $W_{l}^{\min }=64$ and

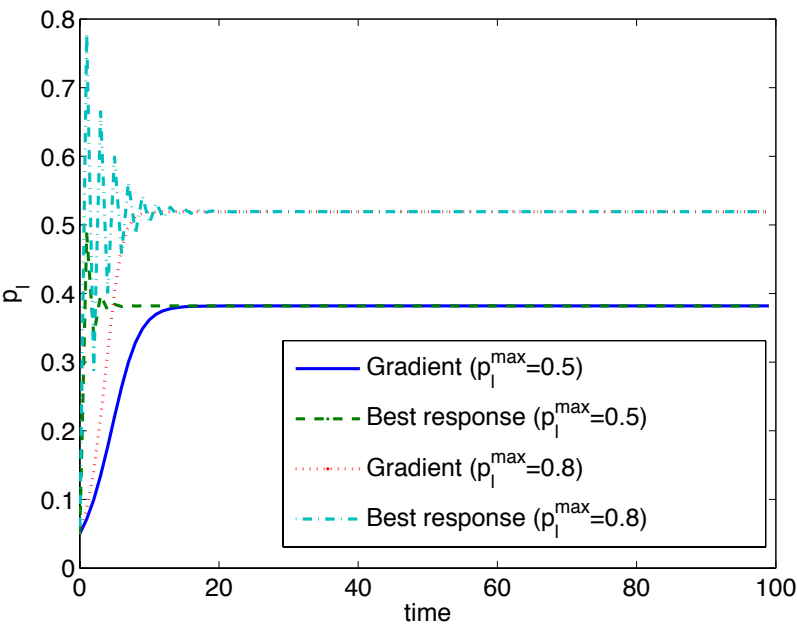

Fig. 6. Comparison of trajectories of $p_{1}(t)$ in a system with two links.

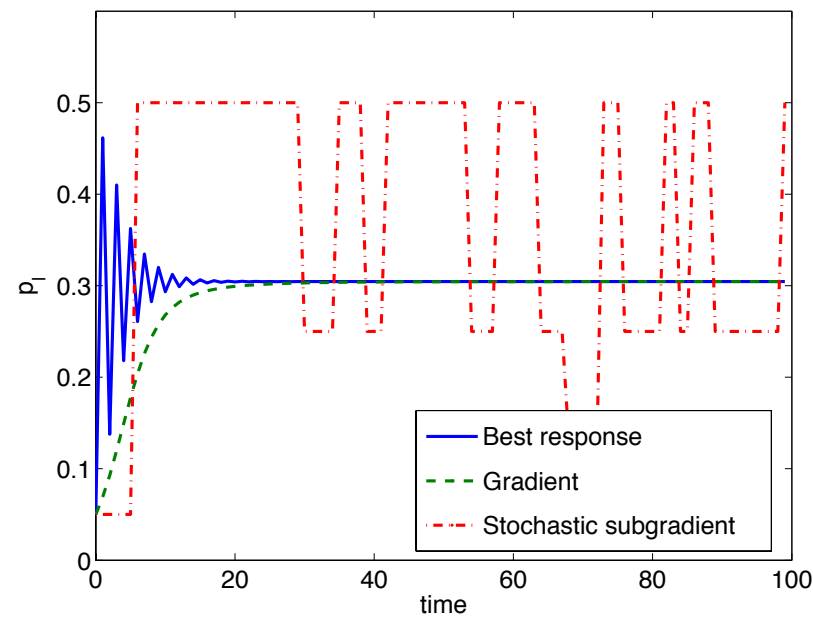

Fig. 7. Comparison of trajectories of $p_{1}(t)$ in the network in Figure 1, with $p_{l}^{\max }=0.5$.

$W_{l}^{\max }=1024$, which correspond to $p^{\min }=2 / 1025$ and $p^{\max }=2 / 65$ in our probabilistic model. In Figure 5, we plot the minimum value of $\beta$ that satisfies condition 3 as a function of the number of interfering links $M_{l}$. It is clear from the figure that any nonnegative value of $\beta$ satisfies condition 3 when $M_{l} \leq 23$. For any $\beta \geq 0.5$, condition 3 is satisfied with $M_{l} \leq 37$, which is large enough even for a dense network. For other physical layer specifications such as Frequency Hopping Spread Spectrum (FHSS) and Direct Sequence Spread Spectrum (DSSS), the minimum contention window sizes are $W_{l}^{\min }=16$ and $W_{l}^{\min }=32$, respectively ([2]). The maximum contention window sizes are the same as in the IR case. As a result, any $\beta \geq 0.5$ satisfies condition 3 when $M_{l} \leq 9$ and $M_{l} \leq 18$, for FHSS and DSSS respectively. For all three physical layer specifications, condition 2 is automatically satisfied in all practical scenarios (i.e., $\beta \geq 0$ and $M_{l} \leq 2099$ ).

\section{NUMERICAL EXAMPLES}

We present numerical results for the non-cooperative game model for MAC protocol. In Figure 6, we consider a network 


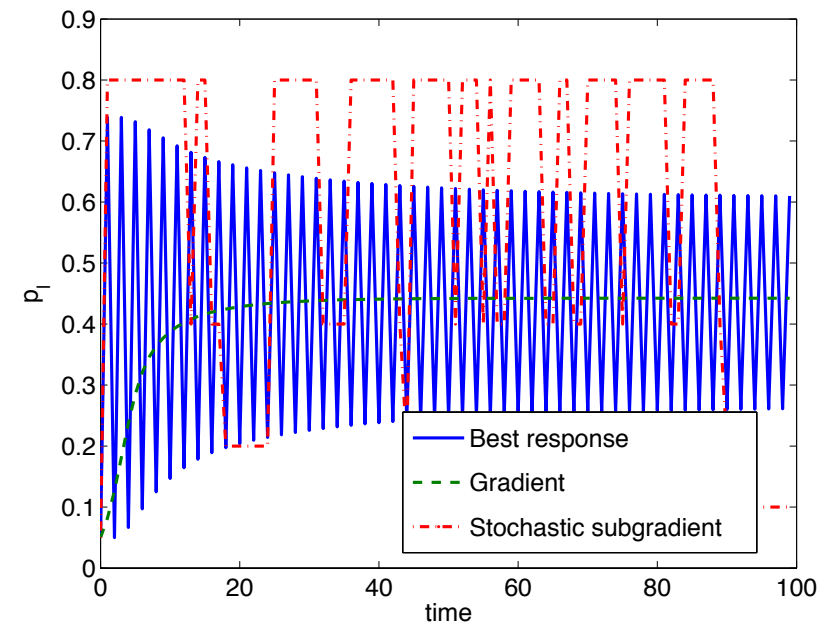

Fig. 8. Comparison of trajectories of $p_{1}(t)$ in the network in Figure 1, with $p_{l}^{\max }=0.8$.

with two links. We first provide the results with $p_{l}^{\max }=0.5$ and $p_{l}^{\max }=0.8$ in the same graph, setting $\beta_{l}=0.5$ and $p_{l}^{\min }=0.05$ for both cases. We compare trajectories of the persistence probability of link $1, p_{1}(t)$, which are obtained by (3), i.e., by gradient updates, and by (6), i.e., by best response. It can in fact be proved that, in the two-link case, the trajectory of the persistence probability obtained by (6) converges to a Nash equilibrium, which is confirmed in this numerical example. The trajectory obtained by (3) converges to the same Nash equilibrium, but more smoothly than that obtained by (6).

In Figures 7 and 8, we consider the network in Figure 1 , which has six logical links, with $\beta_{l}=0.5$ and $p_{l}^{\text {min }}=$ 0.05 , and plot the trajectories of link 1 . In these figures, we also provide trajectories obtained by (2), i.e., by stochastic subgradient. ${ }^{6}$ In Figure 7 , we set $p_{l}^{\max }=0.5$. The figure shows that trajectories obtained by (3) and (6) converge to the same equilibrium, which must be a Nash equilibrium from Theorem 3. ${ }^{7}$ In Figure 8, we set $p_{l}^{\max }=0.8$. The figure shows that the trajectory obtained by (6) oscillates between two values. Indeed, as shown in Theorem 3, in general the EBMAC Game with the best response strategy may not converge to a Nash equilibrium. Furthermore, while the trajectory obtained by gradient method (3) converges and, by Corollary 1 , it indeed converges to a Nash equilibrium, the stochastic subgradient iterations do not converge in this example. In other simulations, we observe that the moving average of the stochastic subgradient updates with a diminishing step-size converges.

In Figures 9 and 10, we consider a two-link topology and compare the attained Nash equilibrium when each link has a different $p_{l}^{\max }$ and a different $\beta_{l}$, respectively. In Figure 9, we set $\beta_{1}=\beta_{2}=0.5$. But link 1 has its maximum persistence probability $p_{1}^{\max }=0.5$ and link 2 has its maximum persistence probability $p_{2}^{\max }=0.5+a$. In Figure 10, we set $p_{1}^{\max }=p_{2}^{\max }=0.5$. But link 1 has $\beta_{1}=0.5$ and link

\footnotetext{
${ }^{6}$ Since $p_{l}(t)$ is a stochastic process in this case, we plot its sample path.

${ }^{7}$ Although not shown in the graph, trajectories of the persistence probabilities of the other links also converge.
}

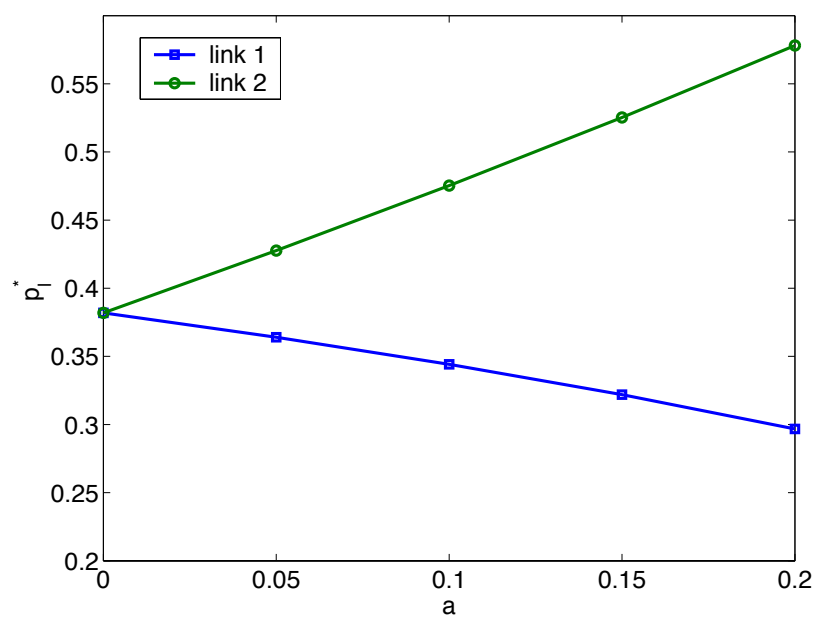

Fig. 9. Comparison of the persistence probability of links that have different $p_{l}^{\max }: \beta_{1}=\beta_{2}=0.5$, but $p_{1}^{\max }=0.5$ and $p_{2}^{\max }=0.5+a$.

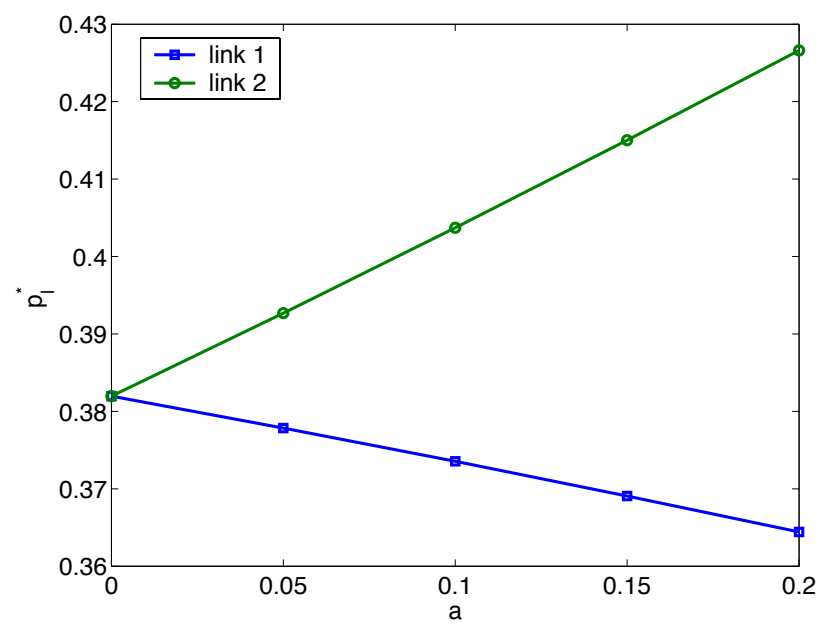

Fig. 10. Comparison of the persistence probability of links that have different $\beta_{l}: p_{1}^{\max }=p_{2}^{\max }=0.5$, but $\beta_{1}=0.5$ and $\beta_{2}=0.5+a$.

2 has $\beta_{2}=0.5+a$. Hence, in both figures, as the value of $a$ gets larger, link 2 updates its persistence probability more aggressively than link 1 . As a consequence, link 2 converges to a higher persistence probability and link 1 to a lower persistence probability, with the difference between the two increasing as the value of $a$ becomes larger. This demonstrates that parameter setting of a link affects not only the performance of the corresponding link but also the performance of other links, causing fairness issues at the Nash equilibrium.

\section{CONCLUSiOnS}

Starting with given MAC protocol specifications, we have reverse-engineered exponential-backoff random access protocols as a non-cooperative game where each link is implicitly maximizing, through a stochastic subgradient update, a quasi-concave utility function in the form of net reward for successful transmission. Due to the lack of proper feedback mechanisms in the current EB protocols, such selfish, local actions are not aligned to maximize the network-wide total utility, nor are they guaranteed to converge, even though a 
Nash equilibrium for the MAC game always exists. We have provided sufficient conditions (on link density and backoff aggressiveness) that guarantee both the uniqueness of Nash equilibrium, then characterized convergence of gradient play with small stepsize, and convergence of the best response strategy. Finally we established the connection between stochastic subgradient and best response for the EB-MAC game.

Our MAC layer reverse-engineering results, together with the recently established reverse-engineering optimization models for TCP and BGP, provide a mathematical foundation for those layers 2-4 protocols that were originally designed based on ad hoc heuristics. Deficiencies of existing MAC protocols revealed through reverse-engineering also motivates forward engineering, where adequate feedback is generated to align selfish utility maximization by each logical link to maximize the social welfare [18].

The formulation and results in this paper can be a basis to further study other properties of EB MAC protocols, such as efficiency loss of the non-cooperative game compared with social welfare maximization. Like the reverse-engineering models of TCP and BGP, there are several simplifying assumptions in our model, notably our focus only on the contention resolution mechanism. A next step is to reverse-engineer carrier-sensing-based (e.g., RTS-CTS) MAC protocols (e.g., CSMA/CA) that consists of both contention avoidance and collision resolution algorithms. Finally, session level stochastic effects need to be incorporated to include the arrival statistics of finite-duration sessions. Then MAC protocols can be analyzed and designed using both stochastic stability results in traditional queuing models and optimality results in the utility maximization models.

\section{ACKNOWLEDGE}

We appreciate the helpful discussions with Steven Low and Lijun Chen at Caltech and Amir Hamed Mohsenian Rad from University of British Columbia.

\section{APPENDIX}

\section{A. Proof of Theorem 1}

Proof: We first obtain the utility function of each link based on the update algorithm in (3). Assuming that there exists an equilibrium with persistence probabilities $\mathbf{p}^{*}$, $\mathbf{p}^{\min }<\mathbf{p}^{*}<\mathbf{p}^{\max }$, then we see from (3) that $\mathbf{p}^{*}$ satisfies the following:

$$
\begin{aligned}
p_{l}^{*}= & p_{l}^{\max } p_{l}^{*} \prod_{n \in L_{t o}^{I}(l)}\left(1-p_{n}^{*}\right)+\beta_{l} p_{l}^{*} p_{l}^{*}\left(1-\prod_{n \in L_{t o}^{I}(l)}\left(1-p_{n}^{*}\right)\right. \\
& +p_{l}^{*}\left(1-p_{l}^{*}\right) .
\end{aligned}
$$

Since each link adjusts its own persistence probability to maximize its utility given persistence probabilities of the other link, from (11) and the first order necessary condition, each link $l$ has its utility function, $U_{l}(\mathbf{p})$, such that

$$
\begin{aligned}
\frac{\partial U_{l}(\mathbf{p})}{\partial p_{l}}= & p_{l}^{\max } p_{l} \prod_{n \in L_{t o}^{I}(l)}\left(1-p_{n}\right) \\
& +\beta_{l} p_{l} p_{l}\left(1-\prod_{n \in L_{t o}^{I}(l)}\left(1-p_{n}\right)\right)+p_{l}\left(1-p_{l}\right)-p_{l} .
\end{aligned}
$$

up to a constant offset, is obtained as

$$
\begin{aligned}
U_{l}(\mathbf{p})= & \frac{1}{2} p_{l}^{\max } \prod_{n \in L_{t o}^{I}(l)}\left(1-p_{n}\right) p_{l}^{2} \\
& +\frac{1}{3} \beta_{l}\left(1-\prod_{n \in L_{t o}^{I}(l)}\left(1-p_{n}\right)\right) p_{l}^{3}-\frac{1}{3} p_{l}^{3} \\
= & p_{l}^{2} \prod_{n \in L_{t o}^{I}(l)}\left(1-p_{n}\right)\left(\frac{1}{2} p_{l}^{\max }\right. \\
& \left.-\frac{1}{3} p_{l}\right)-\frac{1}{3}\left(1-\beta_{l}\right) p_{l}^{3}\left(1-\prod_{n \in L_{t o}^{I}(l)}\left(1-p_{n}\right)\right) \\
= & R\left(p_{l}\right) S(\mathbf{p})-C\left(p_{l}\right) F(\mathbf{p}),
\end{aligned}
$$

where $R\left(p_{l}\right)=p_{l}\left(\frac{1}{2} p_{l}^{\max }-\frac{1}{3} p_{l}\right), C\left(p_{l}\right)=\frac{1}{3}\left(1-\beta_{l}\right) p_{l}^{2}$, $S(\mathbf{p})=p_{l} \prod_{n \in L_{t o}^{I}(l)}\left(1-p_{n}\right)$, and $F(\mathbf{p})=p_{l}(1-$ $\prod_{n \in L_{t o}^{I}(l)}\left(1-p_{n}\right)$.

It can be verified that utility function $U_{l}$ is quasi-concave in $p_{l}$. The action set $A_{l}=\left\{p_{l} \mid p_{l}^{\text {min }} \leq p_{l} \leq p_{l}^{\max }\right\}$ of each link $l$ is a nonempty compact convex subset of Euclidian space, and the utility function $U_{l}$ of each link $l$ is continuous and quasi-concave on $A_{l}$. Hence, by Proposition 20.3 in [13], there exists a Nash equilibrium.

Moreover, from (12), we can easily show that

$$
\frac{\partial U_{l}(\mathbf{p})}{\partial p_{l}}\left\{\begin{array}{l}
>0, \text { if } p_{l}<\frac{p_{l}^{\max } \prod_{n \in L_{t o}^{I}(l)}\left(1-p_{n}\right)}{1-\beta_{l}\left(1-\prod_{n \in L_{t o}^{I}(l)}\left(1-p_{n}\right)\right)} . \\
<0, \text { otherwise }
\end{array}\right.
$$

Hence, we can characterize Nash equilibrium for persistence probabilities of links as

$$
p_{l}^{*}=\frac{p_{l}^{\max } \prod_{n \in L_{t o}^{I}(l)}\left(1-p_{n}^{*}\right)}{1-\beta_{l}\left(1-\prod_{n \in L_{t o}^{I}(l)}\left(1-p_{n}^{*}\right)\right)}, \forall l .
$$

\section{B. Proof of Theorem 3}

Proof: We have

$$
\frac{\partial^{2} U_{l}(\mathbf{p})}{\partial p_{l} \partial p_{k}}= \begin{cases}\prod_{\substack{n \\ n}}\left(1-p_{n}^{I}(l)\left(\beta_{l} p_{l}^{2}-p_{l}^{\max } p_{l}\right), k \in L_{t o}^{I}(l)\right. \\ n \neq k & \text { otherwise }\end{cases}
$$

Since $\beta_{l}<1$ and $p_{l} \leq p_{l}^{\max }$, the utility function is submodular $^{8}$. Moreover, the action set of a link does not depend on the strategies of the other links. Hence, by applying Theorem 5.1 in [16], the proof is completed.

\section{Proof of Lemma 1}

Proof: $\prod_{i \in L_{t o}^{I}(l)}\left(1-p_{i}^{*}\right) \leq 1$. It is easy to check $p_{l}^{*}$ achieves its maximal $p^{\max }$ when $\prod_{i \in L_{t o}^{I}(l)}\left(1-p_{i}^{*}\right)=1$. Therefore $p_{l}^{*} \leq p^{\max }$.

If $p_{l}^{*}=0$, then $p_{i}^{*}=1$ for some $i \in L_{t o}^{I}(l)$. That is impossible as we know $p_{i}^{*} \leq p^{\max }<1$. Hence $p_{l}^{*}>0$.

If $p_{l}^{*}=p^{\max }$, then $\prod_{i \in L_{t o}^{I}(l)}\left(1-p_{i}^{*}\right)=1$. That is again impossible as $p_{i}^{*}>0$. Hence $p_{l}^{*}<p^{\max }$.

${ }^{8}$ If $U_{l}$ is twice differentiable and $\frac{\partial^{2} U_{l}(\mathbf{p})}{\partial p_{l} \partial p_{k}} \leq 0, \forall \mathbf{p} \in \times_{l \in L} A_{l} \forall k \neq l$, then $U_{l}$ is submodular. We refer readers to [15], [16] for more details on submodularity. 


\section{Proof of Theorem 4}

Proof: The best response updating rule is defined as following:

$$
p_{l}(t+1)=p^{\max } \frac{\prod_{i \in L_{t o}^{I}(l)}\left(1-p_{i}(t)\right)}{1-\beta\left(1-\prod_{i \in L_{t o}^{I}(l)}\left(1-p_{i}(t)\right)\right)} .
$$

Its equilibrium is characterized by (7). We now set up uniqueness and convergence together by showing (15) is a contraction mapping. We first cite the following basic theorem [17] that we will use.

Contraction Mapping Theorem. Let $M$ be a complete metric space and $f: M \rightarrow M$ a mapping. Assume there is a constant $k$, where $0 \leq k<1$, such that $d(f(u), f(v)) \leq k d(u, v)$, for all $u, v \in M$; such an $f$ is called a contraction. Then $f$ has a unique fixed point; that is, there exists a unique $u^{*} \in M$. Furthermore, the sequence $u(t+1)=f(u(t))$ converges to the unique fixed point.

Let $M$ be the Euclidean space and consider any vector norm. Let $d($.$) be the induced distance function by the vector$ norm. We have

$$
\begin{aligned}
d(f(u), f(v)) & =\|f(u)-f(v)\| \\
& \leq\left\|\frac{\partial f}{\partial x}\right\|\|(u-v)\|=\left\|\frac{\partial f}{\partial x}\right\| d(u, v) .
\end{aligned}
$$

The matrix norm used here is induced by the vector norm too. The inequality follows from the property of matrix norm. Hence it is clear that if we have the Jocobian $\left\|\frac{\partial f}{\partial x}\right\|<1-\epsilon$ everywhere for some positive $\epsilon$, we can let $k=1-\epsilon<1$ and the Contraction Mapping Theorem applies. ${ }^{9}$

We now derive conditions using $\|.\|_{\infty}$ for (15) to be a contraction map. Its Jacobian $\mathbf{J}$ is defined by

$$
J_{l j}=\frac{\partial p_{l}(t+1)}{\partial p_{j}(t)}
$$

It is straightforward to check

$$
J_{l j}=\left\{\begin{array}{lr}
0, & j \notin L_{t o}^{I}(l) \\
-p^{\max } \frac{(1-\beta) \prod_{i \in L_{t o}^{I}(l), i \neq j}\left(1-p_{i}\right)}{\left(1-\beta\left(1-\prod_{i \in L_{t o}^{I}(l)}\left(1-p_{i}\right)\right)\right)^{2}}, & j \in L_{t o}^{I}(l)
\end{array}\right.
$$

It then follows that

$$
\|\mathbf{J}\|_{\infty}=\max _{l}\left\{p^{\max } \sum_{j \in L_{t o}^{I}(l)} \frac{(1-\beta) \prod_{i \in L_{t o}^{I}(l), i \neq j}\left(1-p_{i}\right)}{\left(1-\beta\left(1-\prod_{i \in L_{t o}^{I}(l)}\left(1-p_{i}\right)\right)\right)^{2}}\right\} .
$$

For any $j \in L_{t o}^{I}(l)$, define

$$
\pi(l, j)=\prod_{i \in L_{t o}^{I}(l), i \neq j}\left(1-p_{i}\right)
$$

and

$$
M(l, j)=\frac{(1-\beta) \prod_{i \in L_{t o}^{I}(l), i \neq j}\left(1-p_{i}\right)}{\left(1-\beta\left(1-\left(1-p_{j}\right) \prod_{i \in L_{t o}^{I}(l), i \neq j}\left(1-p_{i}\right)\right)\right)^{2}} .
$$

We have

$$
M(l, j)=\frac{(1-\beta) \pi(l, j)}{\left(1-\beta\left(1-\left(1-p_{j}\right) \pi(l, j)\right)\right)^{2}}
$$

\footnotetext{
${ }^{9}$ As $\epsilon$ can be arbitrarily small, the later derivation will use 1 instead.
}

and

$$
\frac{d M(l, j)}{d \pi(l, j)}=\frac{(1-\beta)\left(1-\beta-\beta \pi(l, j)\left(1-p_{j}\right)\right)}{\left(1-\beta\left(1-\left(1-p_{j}\right) \pi(l, j)\right)\right)^{3}} .
$$

It then follows that, if $\frac{1-\beta}{\beta\left(1-p_{j}\right)} \leq 1, M(l, j)$ achieves its maximum value of $\frac{1}{4 \beta\left(1-p_{j}\right)}$ when $\pi(l, j)=\frac{1-\beta}{\beta\left(1-p_{j}\right)}$, i.e., $\prod_{i \in L_{t o}^{I}(l)}\left(1-p_{i}\right)=\frac{1-\beta}{\beta}$. If $\frac{1-\beta}{\beta\left(1-p_{j}\right)} \geq 1, M(l, j)$ reaches its maximum value of $\frac{1-\beta}{\left(1-\beta+\beta\left(1-p_{j}\right)\right)^{2}}$ when $\pi(l, j)=1 .^{10}$ Therefore, we conclude that

$$
\begin{aligned}
\|\mathbf{J}\|_{\infty} & =\max _{l}\left\{p^{\max } \sum_{j \in L_{t o}^{I}(l)} M(l, j)\right\} \\
& \leq \max _{l}\left\{\frac{p^{\max }\left|L_{t o}^{I}(l)\right|}{4 \beta\left(1-p^{\max }\right)}\right\} \leq \frac{p^{\max } K}{4 \beta\left(1-p^{\max }\right)} .
\end{aligned}
$$

By assumption in the theorem, we conclude $\|J\|_{\infty}<1$. Hence, (15) is a contraction mapping and both uniqueness and global convergence are guaranteed [17].

\section{E. Derivation of the limit in Footnote 5}

We now show the upperbound of $p^{\max }$ cannot be made independent of $L$ via above method, by deriving an upperlimit considering the system in which each link interfere each other (i.e., $\left.L_{t o}^{I}(l)=E-\{l\}, \forall l\right)$ that takes into account the relation among $M(l, j)$ for different $j$, which has been neglected in previous derivation. We have

$$
\|J\|_{\infty}=p^{\max } \sum_{j \neq l} \frac{(1-\beta) \prod_{i \neq l, j}\left(1-p_{i}\right)}{\left(1-\beta\left(1-\prod_{i \neq l}\left(1-p_{i}\right)\right)\right)^{2}} .
$$

Let $y_{i}=1-p_{i}$, then

$$
\|J\|_{\infty}=p^{\max }(1-\beta) \sum_{j \neq l} \frac{\prod_{i \neq l, j} y_{i}}{\left(1-\beta\left(1-\prod_{i \neq l} y_{i}\right)\right)^{2}} .
$$

We are interested in finding its maximum with constraint $y_{i} \in$ $\left[1-p^{\max }, 1\right]$, it is at least as big as the maximum of $V(y)=$ $p^{\max }(1-\beta) \frac{(L-1) y^{L-2}}{\left(1-\beta+\beta y^{L-1}\right)^{2}}$, where $y \in\left[1-p^{\max }, 1\right]$.

$$
\begin{aligned}
\frac{d V(y)}{d y}= & p^{\max }(1-\beta) \frac{\left(1-\beta+\beta y^{L-1}\right)(L-1)(L-2) y^{L-3}}{\left(1-\beta+\beta y^{L-1}\right)^{3}} \\
& -\frac{2 \beta(L-1)^{2} y^{2 L-4}}{\left(1-\beta+\beta y^{L-1}\right)^{3}}
\end{aligned}
$$

Solving the optimality condition $\frac{d V(y)}{d y}=0$ gives the critical value

$$
y^{c}=\left(\frac{(1-\beta)(L-2)}{\beta L}\right)^{\frac{1}{L-1}} \text {. }
$$

Therefore, if $y^{c}<1-p^{\max }, \max (V(y))=V\left(1-p^{\max }\right)$; if $y^{c}>1,{ }^{11} \max (V(y))=V(1)$; otherwise, $\max (V(y))=$ $V\left(y^{c}\right)$. Imposing $\max (V(y))<1$, we achieve the limit for upperbound for $p^{\max }$ via using contraction mapping and infinity norm.

\footnotetext{
${ }^{10}$ It is interesting to note that for the standard parameter setting $\beta=0.5$, $\frac{1-\beta}{\beta\left(1-p_{j}\right)} \geq 1$ always holds.

${ }^{11}$ This can only happen if $\beta<\frac{L-2}{2 L-2}$, and cannot happen with $\beta=0.5$.
} 


\section{F. Proof of Theorem 5}

Proof: The proof is similar as in [19]. First we observe from (12) that

$$
\begin{aligned}
\frac{\partial U_{l}(\mathbf{p})}{\partial p_{l}} & =\left(\beta_{l}-1\right) p_{l}^{2}+\left(p_{l} p_{l}^{\max }-\beta_{l} p_{l}^{2}\right) \prod_{n \neq l}\left(1-p_{n}\right) \\
& =\left(p_{l} p_{l}^{\max }-\beta_{l} p_{l}^{2}\right) Q(\mathbf{p})
\end{aligned}
$$

where

$$
Q(\mathbf{p})=\frac{p_{l}\left(\beta_{l}-1\right)}{p_{l}^{\max }-\beta_{l} p_{l}}+\prod_{n \neq l}\left(1-p_{n}\right) .
$$

Let us consider the Lyapunov function

$V(\mathbf{p})=\sum_{l} \frac{1-\beta_{l}}{\beta_{l}}\left(p_{l}+\frac{p_{l}^{\max }}{\beta_{l}} \ln \left(p_{l}^{\max }-\beta_{l} p_{l}\right)\right)-\prod_{n \in E}\left(1-p_{n}\right)$,

hence

$$
\nabla_{l} V(\mathbf{p}(t))=Q(\mathbf{p}(t)) .
$$

Also consider a matrix $D(\mathbf{p}):=-\nabla^{2} V(\mathbf{p})$, from [20, Prop. A.25] we have

$$
\|D(\mathbf{p})\|_{2}^{2} \leq\|D(\mathbf{p})\|_{\infty} \cdot\|D(\mathbf{p})\|_{1} \cdot
$$

Since $D(\mathbf{p})$ is symmetric, $\|D(\mathbf{p})\|_{\infty}=\|D(\mathbf{p})\|_{1}$, and hence

$$
\begin{aligned}
\|D(\mathbf{p})\|_{2} \leq & \|D(\mathbf{p})\|_{\infty} \\
= & \max _{l} \sum_{n}[D(\mathbf{p})]_{l, n} \\
= & \max _{l} \sum_{n}\left(\frac{p_{l}^{\max }\left(1-\beta_{l}\right)}{\left(p_{l}^{\max }-\beta_{l} p_{l}\right)^{2}} \mathbf{1}_{\{l=n\}}\right. \\
& \left.+\prod_{n \neq l, j}\left(1-p_{n}\right) \mathbf{1}_{\{l \neq n\}}\right) \\
\leq & \max _{l} \sum_{n}\left(\frac{p_{l}^{\max }\left(1-\beta_{l}\right)}{\left(p_{l}^{\max }-\beta_{l} p_{l}^{\max }\right)^{2}} \mathbf{1}_{\{l=n\}}+\mathbf{1}_{\{l \neq n\}}\right) \\
\leq & \gamma+|E|-1 .
\end{aligned}
$$

Now let us look at the incremental difference of the Lyapunov function,

$$
\begin{aligned}
& V(\mathbf{p}(t+1))-V(\mathbf{p}(t)) \\
= & \nabla V(\mathbf{p}(t)) \cdot(\mathbf{p}(t+1)-\mathbf{p}(t)) \\
& +\frac{1}{2}(\mathbf{p}(t+1)-\mathbf{p}(t))^{T} \cdot \nabla^{2} V(\overline{\mathbf{p}}) \cdot(\mathbf{p}(t+1)-\mathbf{p}(t)) \\
= & \nabla V(\mathbf{p}(t)) \cdot(\mathbf{p}(t+1)-\mathbf{p}(t)) \\
& -\frac{1}{2}(\mathbf{p}(t+1)-\mathbf{p}(t))^{T} \cdot D(\overline{\mathbf{p}}) \cdot(\mathbf{p}(t+1)-\mathbf{p}(t)) \\
\geq & \nabla V(\mathbf{p}(t)) \cdot(\mathbf{p}(t+1)-\mathbf{p}(t)) \\
& -\frac{\gamma+|E|-1}{2}\|\mathbf{p}(t+1)-\mathbf{p}(t)\|^{2} \\
= & \sum_{l}\left(\nabla_{l} V(\mathbf{p}(t))\left(p_{l}(t+1)-p_{l}(t)\right)\right. \\
& \left.-\frac{\gamma+|E|-1}{2}\left(p_{l}(t+1)-p_{l}(t)\right)^{2}\right)
\end{aligned}
$$

where $\overline{\mathbf{p}}$ is some convex combination of $\mathbf{p}(t)$ and $\mathbf{p}(t+1)$.

We know that $p_{l}(t+1) \leq p_{l}^{\max }$ as long as $\kappa \leq 1$. It is clear that if $p_{l}(t+1)=p_{l}(t)$, (19) equals 0 . Otherwise, we need to consider the following two cases:
1) $p_{l}(t+1) \in\left(p_{l}^{\min }, p_{l}^{\max }\right]$. We have

$$
\begin{aligned}
& \nabla_{l} V(\mathbf{p}(t))\left(p_{l}(t+1)-p_{l}(t)\right) \\
& -\frac{\gamma+|E|-1}{2}\left(p_{l}(t+1)-p_{l}(t)\right)^{2} \\
= & Q(\mathbf{p}(t))\left(\kappa\left(p_{l} p_{l}^{\max }-\beta_{l} p_{l}^{2}\right) Q(\mathbf{p})\right) \\
& -\frac{\gamma+|E|-1}{2}\left(\kappa\left(p_{l} p_{l}^{\max }-\beta_{l} p_{l}^{2}\right) Q(\mathbf{p})\right)^{2} \\
\geq & \left(1-\kappa\left(p_{l} p_{l}^{\max }-\beta_{l} p_{l}^{2}\right) \frac{\gamma+|E|-1}{2}\right) \\
& \kappa\left(p_{l} p_{l}^{\max }-\beta_{l} p_{l}^{2}\right) Q^{2}(\mathbf{p}(t)) \\
\geq & \left(1-\kappa\left(p_{l}^{\max }\right)^{2} \frac{\gamma+|E|-1}{2}\right) \\
& \kappa\left(p_{l} p_{l}^{\max }-\beta_{l} p_{l}^{2}\right) Q^{2}(\mathbf{p}(t)) \\
\geq 0 . &
\end{aligned}
$$

The last inequality is due to the fact that $\kappa \leq$ $\frac{2}{\min _{l}\left(p_{l}^{\max }\right)^{2}(\gamma+|E|-1)}$.

2) $p_{l}(t+1)=p_{l}^{\min }$. This implies that $0>p_{l}^{\min }-p_{l}(t) \geq$ $\kappa\left(p_{l}(t) p_{l}^{\max }-\beta_{l} p_{l}^{2}(t)\right) Q(\mathbf{p}(t))$, and

$$
\begin{aligned}
& \nabla_{l} V(\mathbf{p}(t))\left(p_{l}(t+1)-p_{l}(t)\right) \\
& -\frac{\gamma+|E|-1}{2}\left(p_{l}(t+1)-p_{l}(t)\right)^{2} \\
& =\left(\frac{Q(\mathbf{p}(t))}{p_{l}^{\min }-p_{l}(t)}-\frac{\gamma+|E|-1}{2}\right)\left(p_{l}^{\min }-p_{l}(t)\right)^{2} \\
& \geq\left(\frac{1}{\kappa\left(p_{l} p_{l}^{\max }-\beta_{l} p_{l}^{2}\right)}-\frac{\gamma+|E|-1}{2}\right)\left(p_{l}^{\min }-p_{l}(t)\right)^{2} \\
& \geq 0
\end{aligned}
$$

Combining the above analysis, we always have $V(\mathbf{p}(t+1))-V(\mathbf{p}(t)) \geq 0$, i.e., $V(\mathbf{p})$ will keep increasing till the system reaches a fixed point of equation (9). Since the strategy space $\Pi_{l}\left[p_{l}^{\min }, p_{l}^{\max }\right]$ is an invariant set from the definition of (9) and there is a unique Nash Equilibrium (which is the unique fixed point of (9)) by assumption, we have proven the global convergence of (9).

\section{G. Proof of Theorem 6}

Proof: The proof relies on Theorem 6.2 in [21], a variation of which is stated below.

Stochastic Subgradient Convergence Theorem. Consider the maximization of a concave continuous one-dimensional function $F(x)$ in $x \in[a, b]$, and let $X^{*}$ be a set of optimal solutions. Consider the following stochastic subgradient projection method:

$$
x(t+1)=\max \{a, \min \{b, x(t)+s(t) \xi(t)\}\}, t=0,1, \ldots
$$

$$
\begin{aligned}
& F\left(x^{*}\right)-F(x(t)) \\
\leq & E\{\xi(t) \mid x(0), \cdots, x(t)\}\left(x^{*}-x(t)\right)+\gamma_{0}(t),
\end{aligned}
$$

where $\gamma_{0}(t)$ may depend on $(x(0), \cdots, x(t)), x^{*} \in X^{*}$, and $s(t)$ is the step size that satisfies 


$$
\begin{gathered}
s(t) \geq 0, \sum_{t=0}^{\infty} s(t)=\infty \\
\sum_{t=0}^{\infty} E\left\{s(t)\left|\gamma_{0}(t)\right|+s^{2}(t)|\xi(t)|^{2}\right\}<\infty .
\end{gathered}
$$

Then $\lim x(t) \in X^{*}$ with probability 1 .

For our proof, we map the elements of the updates in (10) into the elements of the algorithm in $(20,21,22)$. First note that $p_{l}(t+1) \leq p_{l}^{\max }$ when $\kappa(t) \leq 1$. Define

$$
p_{l}^{\text {reflex }}=p^{\max } \frac{\Pi_{n \in L_{t o}^{I}(l)}\left(1-p_{n}\right)}{2\left(1-\beta\left(1-\Pi_{n \in L_{t o}^{I}(l)}\left(1-p_{n}\right)\right)\right)} .
$$

It can be verified that $U_{l}\left(p_{l}\right)$ is strictly concave in $p_{l} \in$ $\left[p_{l}^{\text {reflex }}, p^{\max }\right]$ for fixed $p_{-l}$. Also the unique maximizer of $U_{l}\left(p_{l}\right)$ is $p_{l}^{B R}=2 p_{l}^{\text {reflex }}$. It can be further shown that under conditions 2 and 3 in the theorem, $\tilde{p}_{l}^{\text {min }}$ satisfies

$$
\max \left\{p^{\min }, p_{l}^{\text {reflex }}\right\} \leq \tilde{p}_{l}^{\min } \leq p_{l}^{B R} \leq p^{\max }
$$

for any feasible value $p_{n}\left(n \in L_{t o}^{I}(l)\right)$. These enable us to establish the following mappings: $p_{l} \rightarrow x, U_{l}\left(p_{l}\right) \rightarrow F(x)$, $\left[\tilde{p}_{l}^{\min }, p^{\max }\right] \rightarrow[a, b]$ and $\left\{p_{l}^{B R}\right\} \rightarrow X^{*}$. Here $p_{l}^{B R}$ is the best response solution as in (6).

Now we map $v_{l}(t)$ into $\xi(t)$. Since

$$
\begin{aligned}
E\left\{v_{l}(t) \mid p_{l}(0), \cdots, p_{l}(t)\right\} & =E\left\{v_{l}(t) \mid p_{l}(t)\right\} \\
& =\left.\frac{\partial U_{l}\left(p_{l}\right)}{\partial p_{l}}\right|_{p_{l}=p_{l}(t)},
\end{aligned}
$$

inequality $(21)$ is satisfied with $\gamma_{0}(t)=0$. Finally, $(22)$ is satisfied under condition 1 and the fact that $\left|v_{l}(t)\right|^{2} \leq$ $\left(p^{\max }\right)^{2}$. All the conditions of the Stochastic Subgradient Convergence Theorem are satisfied, and $p_{l}(t)$ converges to the best response solution with probability 1 .

\section{REFERENCES}

[1] A. B. MacKenzie and S. B. Wicker, "Stability of multipacket slotted Aloha with selfish users and perfect information," in IEEE INFOCOM, 2003, pp. 1583-1590.

[2] G. Bianchi, "Performance analysis of the IEEE 802.11 distributed coordination function," IEEE J. Sel. Area Comm., vol. 18, no. 3, pp. 535-547, March 2000.

[3] C. Yuen and P. Marbach, "Price-based rate control in random access networks," IEEE/ACM Trans. on Networking, vol. 13, no. 5, pp. 10271040, December 2005.

[4] J.-W. Lee, M. Chaing, and A. R. Calderbank, "Utility-optimal medium access control reverse and forward engineering," in IEEE INFOCOM, 2006.

[5] S. H. Low, "A duality model of TCP and queue management algorithms," IEEE/ACM Trans. on Networking, vol. 11, no. 4, pp. 525-536, August 2003.

[6] R. Srikant, The mathematics of Internet congestion control. Birkhauser, 2004.

[7] T. G. Griffin, F. B. Shepherd, and G. Wilfong, "The stable path problem and interdomain routing," IEEE/ACM Trans. on Networking, vol. 10, no. 2, pp. 232-243, April 2002.

[8] R. J. La and V. Anantharam, "Utility-based rate control in the Internet for elastic traffic," IEEE/ACM Trans. on Networking, vol. 10, no. 2, pp. 272-286, April 2002.

[9] F. P. Kelly, A. K. Maulloo, and D. K. H. Tan, "Rate control in communication networks: shadow prices, proportional fairness and stability," Journal of the Operational Research Society, vol. 49, no. 3, pp. 237252, March 1998.
[10] S. H. Low and D. E. Lapsley, "Optimization flow control-I: basic algorithm and convergence," IEEE/ACM Trans. on Networking, vol. 7, no. 6, pp. 861-874, December 1999.

[11] J. Mo and J. Walrand, "Fair end-to-end window-based congestion control," IEEE/ACM Trans. on Networking, vol. 8, no. 5, pp. 556-567, October 2000.

[12] T. Moscibroda and R. Wattenhofer, "The complexity of connectivity in wireless networks," in IEEE INFOCOM, 2006.

[13] M. J. Osborne and A. Rubinstein, A course in game theory. MIT Press, 1994.

[14] J. R. Brige and F. Loubeaux, Introduction to Stochastic Programming. Springer, 1997.

[15] D. M. Topkis, "Equilibrium points in nonzero-sum n-person submodular games," SIAM Journal of Control and Optimization, vol. 17, no. 6, pp. 773-787, November 1979.

[16] D. D. Yao, "S-modular games, with queueing applications," Queueing Systems, vol. 21, pp. 449-475, 1995.

[17] R. Abraham, J. Marsden, and T. Ratiu, Manifolds, tensor analysis, and applications. Springer-Verlag, 1988.

[18] J.-W. Lee, M. Chiang, and A. R. Calderbank, "Utility-optimal randomaccess control," to appear in IEEE Trans. on Wireless Commun., 2007.

[19] L. Chen, S. H. Low, and J. C. Doyle, "Random access game and medium access control design," submitted for publication, 2006.

[20] D. P. Bertsekas and J. N. Tsitsiklis, Parallel and Distributed Computation: numerical methods. Prentice Hall, 1989.

[21] Y. Ermoliev and R.-B. Wets, Numerical Techniques for Stochastic Optimization. Springer-Verlag, 1980.

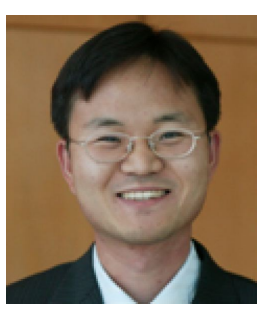

Jang-Won Lee (S' 02 - M' 04) received his B.S. degree in Electronic Engineering from Yonsei University, Seoul, Korea in 1994, M.S. degree in Electrical Engineering from Korea Advanced Institute of Science and Technology (KAIST), Taejon, Korea in 1996, and Ph.D. degree in Electrical and Computer Engineering from Purdue University, West Lafayette, IN, USA in 2004. In 1997-1998, he was employed with Dacom R\&D Center, Taejon, Korea. In 2004-2005, he was a Postdoctoral Research Associate in the Department of Electrical Engineering at Princeton University, Princeton, NJ, USA. Since September 2005, he has been an assistant professor in the School of Electrical and Electronic Engineering at Yonsei University, Seoul, Korea. His research interests include resource allocation, QoS and pricing issues, optimization, and performance analysis in communication networks.

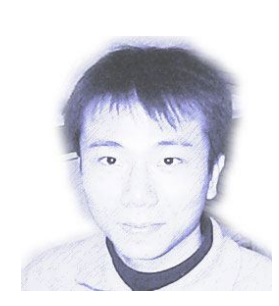

Ao Tang (S' 01 - M' 07) received his B.E. (Hon.) and M.E. in electronics engineering from Tsinghua University, and his Ph.D. in electrical engineering with a minor in applied and computational mathematics from the California Institute of Technology (Caltech), in 1999, 2001, and 2006, respectively.

$\mathrm{He}$ is currently a Junior Fellow in the Social and Information Sciences Laboratory (SISL) at Caltech, where his research interests include control and optimization of communication networks; interconnected dynamical systems; Microeconomics, Game theory and their applications.

Dr.Tang was the recipient of the 2002 Outstanding Master Thesis Award from Tsinghua University, the 2004 National Award for Outstanding Oversea Students from P.R.China, and the 2006 George B. Danzig Best Dissertation Award from INFORMS. 


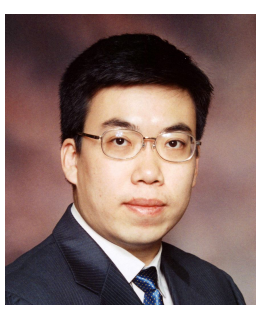

Jianwei Huang ( $S^{\prime} 00-M^{\prime}$ 06) is a Postdoctoral Research Associate in the Department of Electrical Engineering at Princeton University. He received the B.S. degree in Electrical Engineering from Southeast University (Nanjing, China) in 2000, M.S. and Ph.D. degrees in Electrical and Computer Engineering from Northwestern University in 2003 and 2005, respectively. In 2004 and 2005, he worked in the Network Advanced Technology Group at Motorola, both as a full time summer intern and a part time researcher. In 1999, he worked as a summer intern in the Department of Change Management at GKN Westland Aerospace Co. Ltd. His main research interests lie in the area of communications and networking, with specific areas including cognitive radio networks, wideband OFDM and CDMA systems, wireless medium access control, multimedia communications, cooperative communications, and wired DSL broadband access networks.

Dr. Huang is an Associate Editor of Elsevier Journal of Computer \& Electrical Engineering from 2007, the Lead Guest Editor of the special issue of IEEE Journal of Selected Areas in Communications on Game Theory in Communication Systems, the Lead Guest Editor of the special issue of Journal of Advances in Multimedia on Collaboration and Optimization in Multimedia Communications, and a Guest Editor of the special issue of Journal of Advances in Multimedia on Cross-layer Optimized Wireless Multimedia Communications. He is the recipient of a 2001 Walter P. Murphy Fellowship at Northwestern University and a 1999 Chinese National Excellent Student Award.

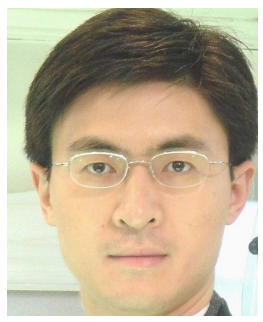

Mung Chiang ( $\mathrm{S}^{\prime} 00-\mathrm{M}^{\prime}$ 03) is an Assistant Professor of Electrical Engineering and an affiliated faculty of Applied and Computational Mathematics and of Computer Science at Princeton University. He received the B.S. (Honors) in Electrical Engineering and Mathematics, M.S. and Ph.D. degrees in Electrical Engineering from Stanford University. Professor Chiang conducts research in the areas of nonlinear optimization of communication systems, theoretical foundation of network architectures, algorithms in broadband access networks, and stochastic models of communications. He has been awarded as a Hertz Foundation Fellow, and received Stanford University School of Engineering Terman Award, SBC Communications New Technology Introduction Contribution Award, NSF CAREER Award, ONR Young Investigator Award, and Princeton University Howard B. Wentz Junior Faculty Award. His Jan. 2005 IEEE JSAC paper becomes the Fast Breaking Paper in Computer Science in 2006 according to ISIs citation frequency, and he co-authored the best student paper at IEEE Globecom 2006.

Professor Chiang is the Lead Guest Editor of the Special Issue of IEEE Journal of Selected Areas in Communications on Nonlinear Optimization of Communication Systems, a Guest Editor of the Joint Special Issue of IEEE Transactions on Information Theory and IEEE/ACM Transactions on Networking on Networking and Information Theory, an Editor of IEEE Transactions on Wireless Communications, a Program Co-Chair of the 38th Conference on Information Sciences and Systems, and a co-editor of the new Springer book series on Optimization and Control of Communication Systems.

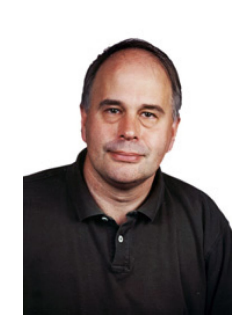

A. Robert Calderbank (M' 89 - SM' 97 - F' 98) received the BSc degree in 1975 from Warwick University, England, the MSc degree in 1976 from Oxford University, England, and the PhD degree in 1980 from the California Institute of Technology, all in mathematics.

$\mathrm{He}$ is currently Professor of Electrical Engineering and Mathematics at Princeton University where he directs the Program in Applied and Computational Mathematics. Dr. Calderbank joined Bell Telephone Laboratories as a Member of Technical Staff in 1980, and retired from AT\&T in 2003 as Vice President of Research. Dr. Calderbank has research interests that range from algebraic coding theory and quantum computing to the design of wireless and radar systems.

Dr. Calderbank served as Editor in Chief of the IEEE TRANSACTIONS ON INFORMATION THEORY from 1995 to 1998, and as Associate Editor for Coding Techniques from 1986 to 1989 . He was a member of the Board of Governors of the IEEE Information Theory Society from 1991 to 1996. Dr. Calderbank was honored by the IEEE Information Theory Prize Paper Award in 1995 for his work on the Z4 linearity of Kerdock and Preparata Codes (joint with A.R. Hammons Jr., P.V. Kumar, N.J.A. Sloane, and P. Sole), and again in 1999 for the invention of space-time codes (joint with V.Tarokh and N. Seshadri). He is a recipient of the IEEE Millennium Medal, and was elected to the National Academy of Engineering in 2005. 